\title{
Preparation \& Microscopic Characterization of Non-Chrome Chemical Conversion Film in Aluminum Surface
}

\author{
Zemin Chen ${ }^{1}$, Pin $\mathrm{Lu}^{2 *}$, Jianzhong $\mathrm{Xu}^{2}$, Yanzun Yang ${ }^{1}$ \\ ${ }^{1}$ School of Chemistry and Material Science, Langfang Normal College, Langfang, China \\ ${ }^{2}$ College of Chemistry and Environmental Science, Hebei University, Baoding, China \\ Email: *lupin421@163.com
}

Received January 5, 2013; revised February 10, 2013; accepted February 26, 2013

\begin{abstract}
A chromium-free environmental protection aluminum surface treatment technology was developed by theoretical analysis and a large number of experiments. Add zirconium ions and cerium ions to the treatment solution, besides adding fluoride, aluminum and hydrogen peroxide, etc. According to the orthogonal test obtained a non-chromate film-formation process of environmental friendly aluminum. The characterization methods including SEM, XPS and XRD were applied to study and analyze the morphology, composition, phase, and corrosion resistance of phosphate film, then discussed the film-forming reaction mechanism. Results showed that chemical conversion film formed on the aluminum surface was uniform, compact and stronger anti-corrosion could replace the traditional, more toxic chromate conversion film.
\end{abstract}

Keywords: Non-Chrome Chemical Conversion Film; Microscopic Characterization; Aluminum Surface

\section{Introduction}

With small density, easy processing and high intension ability, aluminum and its alloy have played a more and more important role in real life. However, it has disadvantages such as low hardness, poor wear resistance and susceptible to intergranular corrosion [1], in order to improve these deficiencies, the aluminum chemical conversion film often formed on the surface layer to obtain high-quality coating, and treatment before processing. At present the chemical conversion film of aluminum mostly use chromium compounds with highly toxic [2-5]. Mainly speaking, the study of environmentally friendly non-chrome conversion film process technology has two directions, one is the zirconium series contained titanium, zirconium and fluoride non-chrome system; the other is with cerium conversion film as the rare earth conversion film [6-8]. Though these processes have overcome the deficiencies of the traditional processes, but the pretreatment processes are complicated. Therefore developing a non-chrome chemical conversion film process of low cost, easy to operate, and environmental friendly has been to be solved desirably in pre-treatment system of painting industry.

${ }^{*}$ Corresponding author.

\section{Experiment}

\subsection{Preparation of Film-Forming Agent}

All used reagents were analytically pure chemicals and secondary distilled water was used to prepare all the solutions.

In the plastic beaker join sulfate $(1: 2,10.2 \mathrm{~mL})$, cerium sulfate $(3.6 \mathrm{~g})$, alum stir $(1.8 \mathrm{~g})$, zirconium dichloride $(4.8 \mathrm{~g})$, potassium fluotitanate $(1.8 \mathrm{~g})$ and hydrogen peroxide $(1: 2,4.50 \mathrm{~mL}$ ) in order (each sample was stirred to dissolve before adding the next). Finally, set the volume with distilled water to $300 \mathrm{~mL}$.

\subsection{Process Specimen}

The aluminum specimens $(50 \times 25 \times 0.3 \mathrm{~mm})$ completely immersion into degreasing rust remover LS-416 (Haihuan Chemical Co. Ltd., Langfang) at the room temperature. Take the aluminum surface without water droplets as the standard of removing oil pollution and rust pollution cleanlily.

After remove cleanly, wash the aluminum specimens with tap water, and then wash them three times with distilled water. Then immersion them into film-forming agent in $40^{\circ} \mathrm{C}$ constant temperature water bath for $20 \mathrm{~min}$, and dry by airing after coating. 


\section{Results and Discussion}

\subsection{XRD Analysis}

In Figure 1, there was not evident peak, which indicating that the oxide film formed was amorphous. But in the SEM Figure 2(a) we can see a spherical crystal packing in the film surface, in the XRD spectra there is only one broad diffraction peak. I think this is due to the chemical conversion film began to form the crystals in the formation process, but the larger atomic radius of $\mathrm{Zr}$, resulting in a larger crystal lattice distortion. This undermines the orderly crystal structure, and so formed the long-range disorder, short-range order of the amorphous structure.

\subsection{XPS Analysis}

Figure 2(a) showed that the formed non-chrome conversion film was a dense amorphous film, and the amorphous film presents plate and cracks. It is found that the adhesion of former coating is higher than the latter's in coating experiments. This illustrated that the development process is greater improvement both on microcosmic structure of conversion coating and membrane performance.

\subsection{XPS Spectra Analysis}

The XRD patterns of conversion film shown in Figure 3. There are several major peaks including boron, oxygen, aluminum, zirconium and cerium, and the maximum peak is aluminum peak which is formed by host, the rest of the several elements are the membrane material.

Zirconium has two peaks: $\mathrm{Zr}(\mathrm{IV})$ into the peak of the strong peaks, indicating that the film $\mathrm{Zr}$ (IV) compounds account for a large proportion, and $\mathrm{Zr}$ (II) ions are also involved in the film, yet low levels. In addition there is no fluorine peak showed that fluoride is not film-forming material. Figure 3 illustrated that conversion coating composition is about $\mathrm{Ce}: \mathrm{Zr}: \mathrm{B}=1: 5: 4$, so we inferred that the composition of the new conversion film was $\mathrm{Ce}_{3}\left(\mathrm{BO}_{3}\right)_{4} \cdot 2 \mathrm{Zr}_{3}\left(\mathrm{BO}_{3}\right)_{4} \cdot 3\left(\mathrm{Zr} \cdot \mathrm{ZrO} \cdot \mathrm{ZrO}_{2} \cdot \mathrm{H}_{2} \mathrm{O}\right)$.

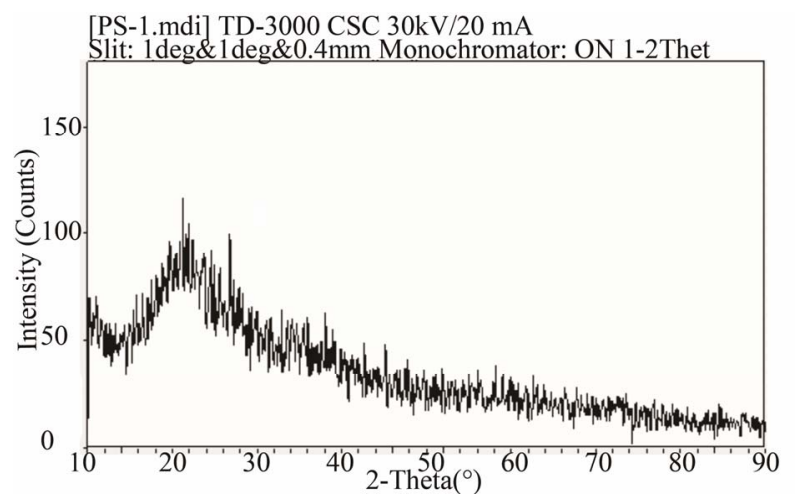

Figure 1. X-diffraction patterns of conversion film.

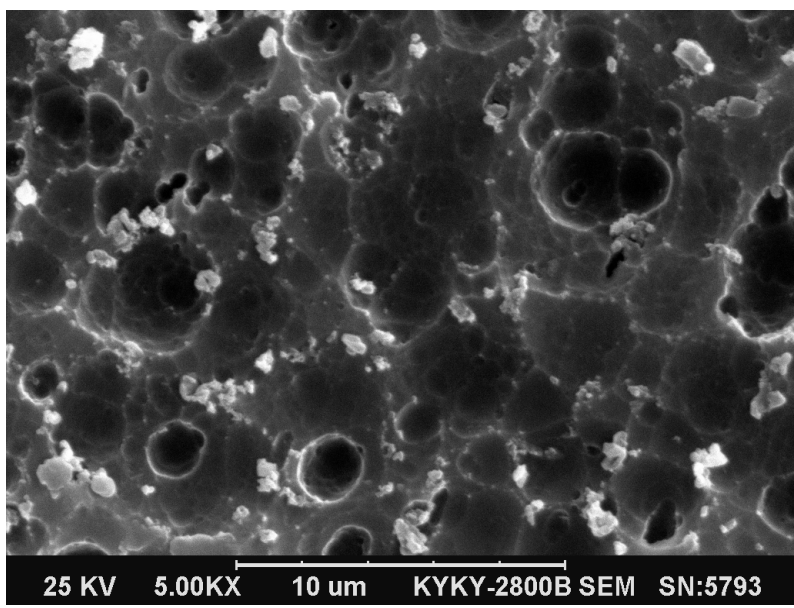

(a)

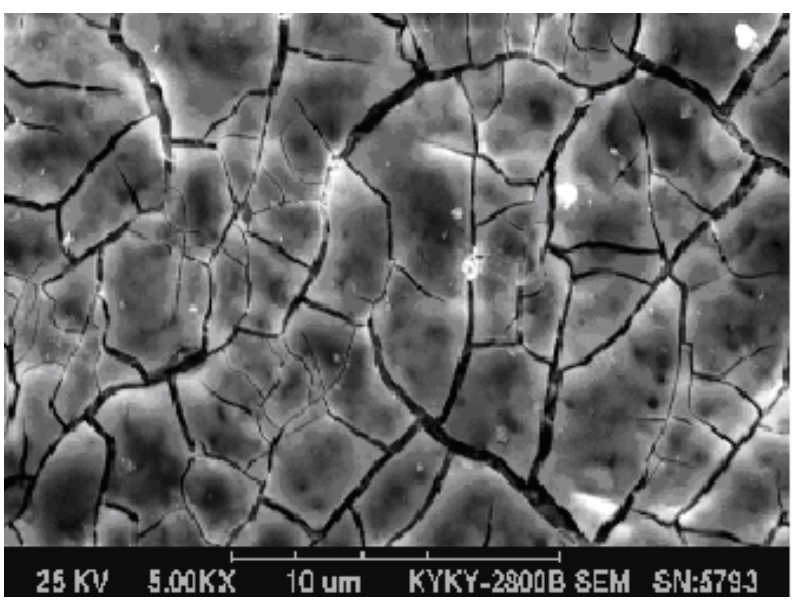

(b)

Figure 2. (a) and (b) SEM images for (a) new conversion coating and (b) chromium conversion coating sample prepared for 20 min of immersion time at RT.

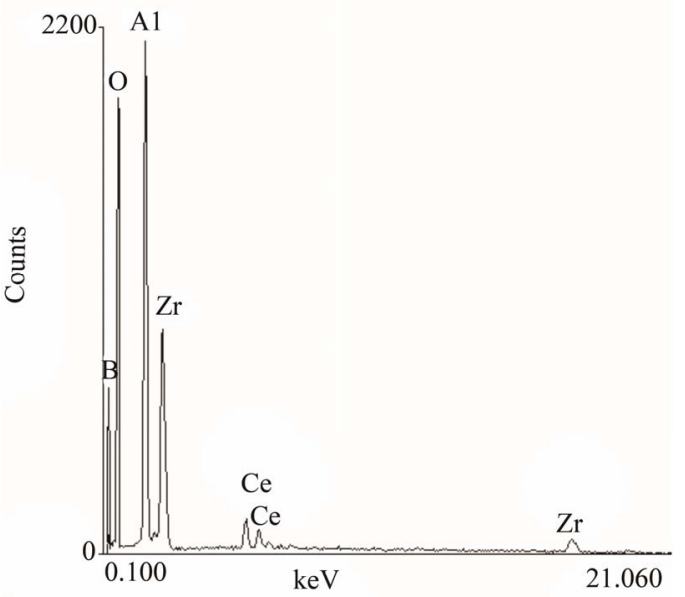

Figure 3. XPS of new conversion film. The prepared sample cut into the specifications of $5 \mathrm{~mm} \times 5 \mathrm{~mm}$, then under the $\mathrm{AV}=25 \mathrm{KeV}, \mathrm{LT}=100 \mathrm{~s}$ conditions $(\mathrm{KYKY}-2800 \mathrm{~B})$ for XPS scan. 


\subsection{Mechanism of Film Formation Film}

The fluoride borate reaction occurs in film-forming agent as follows:

$$
\begin{aligned}
& \mathrm{BF}^{4-} \rightarrow \mathrm{BF}_{3}+\mathrm{F}^{-} \\
& \mathrm{BF}_{3}+3 \mathrm{H}_{2} \mathrm{O} \rightarrow \mathrm{H}_{3} \mathrm{BO}_{3}+3 \mathrm{HF}
\end{aligned}
$$

Fluoride can corrosion and dissolution the aluminum base metal in acid solution $(\mathrm{pH}=3-5)$, and promote the aluminum surface activation.

$$
\begin{aligned}
& 2 \mathrm{Al}+6 \mathrm{H}^{+} \rightarrow 2 \mathrm{Al}^{3+}+3 \mathrm{H}_{2} \uparrow \\
& \mathrm{Al}^{3+}+6 \mathrm{~F}^{-} \rightarrow \mathrm{AlF}_{6}^{3-}
\end{aligned}
$$

The replacement reaction of activated aluminum is as following:

$$
4[\mathrm{Al}]+3 \mathrm{Zr}^{4+} \rightarrow 4 \mathrm{Al}^{3+}+3 \mathrm{Zr}
$$

Because of the chemical activity, zirconium deposited on the film can be oxidized, then protect the film surface.

$2 \mathrm{Zr}+\mathrm{O}_{2} \rightarrow 2 \mathrm{ZrO}$

Owing to the acidity reducing of the metal solution interface, the local $\left[\mathrm{OH}^{-}\right]$concentration transiently increased in this interface area, the reaction is as follows:

$$
\begin{aligned}
& \mathrm{Zr}^{4+}+4 \mathrm{OH}^{-} \rightarrow \mathrm{Zr}(\mathrm{OH})_{4} \downarrow \\
& \mathrm{Zr}(\mathrm{OH})_{4} \rightarrow \mathrm{ZrO}_{2} \cdot \mathrm{H}_{2} \mathrm{O}+\mathrm{H}_{2} \mathrm{O}
\end{aligned}
$$

The theoretical analysis shows that the film is combination of $\mathrm{Zr} \cdot \mathrm{ZrO} \cdot \mathrm{ZrO}_{2} \cdot \mathrm{H}_{2} \mathrm{O}$.

\section{Conclusions}

In this paper, zirconium fluoride and cerium sulfate were used as the main material, the non-chrome conversion film on the aluminum surface was formed by impregnation method. The treatment solution with steady performance, simple operation process and environmental protection could be considered for industrial production.

A preliminary discussion is made on the reaction mechanism of the reaction process, and determined the chemical composition of conversion film.

\section{REFERENCES}

[1] S. de Souza, D. S. Yoshikawa, W. A. S. Izaltino, S. L. Assis and I. Costa, "Self-Assembling Molecules as Corrosion Inhibitors for 1050 Aluminum," Surface and Coatings Technology, Vol. 204, No. 20, 2010, pp. 3238-3242. doi:10.1016/j.surfcoat.2010.03.021

[2] I. Van Roy, H. Terryn and G. Goeminne, "Study of the phosphating Treatment of Aluminium Surfaces: Role of the Activating Process," Colloids and Surfaces A: Physicochemical and Engineering Aspects, Vol. 136, No. 1-2, 1998, pp. 89-96. doi:10.1016/S0927-7757(97)00254-9

[3] A. S. Hamdy, "A Clean Low Cost Anti-Corrosion Molybdate Based Nano-Particles Coating for Aluminum Alloys," Progress in Organic Coatings, Vol. 56, No. 2-3, 2006, pp. 146-150. doi:10.1016/j.porgcoat.2006.03.002

[4] F. Andreatta, P. Aldighieri, L. Paussa, R. Di Maggio, S. Rossi and L. Fedrizzi, "Electrochemical Behaviour of $\mathrm{ZrO}_{2}$ Sol-Gel Pre-Treatments on AA6060 Aluminium Alloy," Electrochimica Acta, Vol. 52, 2007, pp. 7545-7555. doi:10.1016/i.electacta.2006.12.065

[5] Z.-M. Chen, X.-Y. Chen, Y.-Z. Yang, et al., "Aluminum Surface Membrane of Non-Chrome Chemical Conversion Film-Forming Process," Materials Protection, Vol. 3, 2008, pp. $45-46$.

[6] C. Spadaro, C. Dispenza and C. Sunseri, "The influence of the Nature of the Surface Oxide on the Adhesive Fracture Energy of Aluminium-Bonded Joints as Measured by T-Peel Tests," International Journal of Adhesion and Adhesives, Vol. 28, No. 4-5, 2008, pp. 211-221. doi:10.1016/j.ijadhadh.2007.04.001

[7] A. S. Hamdy, "Corrosion Protection of Aluminum Composites by Silicate/Cerate Conversion Coating," Surface and Coatings Technology, Vol. 200, No. 12-13, 2006, pp. 3786-3792. doi:10.1016/j.surfcoat.2005.03.012

[8] S. L. Zhang, "Study on Phosphating Treatment of Aluminum Alloy: Role of Yttrium Oxide," Journal of Rare Earths, Vol. 27, No. 3, 2009, pp. 469-473. doi:10.1016/S1002-0721(08)60271-9 